\title{
Gauß diagram sums on almost positive knots
}

\author{
A. Stoimenow
}

\begin{abstract}
Using the Fiedler-Polyak-Viro Gauß diagram formulae we study the Vassiliev invariants of degree 2 and 3 on almost positive knots. As a consequence we show that the number of almost positive knots of a given genus or unknotting number grows polynomially in the crossing number, and also recover and extend, inter alia to their untwisted Whitehead doubles, previous results on the polynomials and signatures of such knots. In particular, we prove that there are no achiral almost positive knots and classify all almost positive diagrams of the unknot. We give an application to contact geometry (Legendrian knots) and property $P$.
\end{abstract}

\section{Introduction}

The Thurston-Bennequin invariant of Legendrian knots came to prominence when in 1983 Bennequin [Ben83] made use of it to discover a non-standard contact structure on the real space. His proof was based on an inequality, now named after him [Ben83, Theorem 3], estimating this invariant in the standard contact space in terms of the genus of the underlying topological knot. This inequality, unfortunately, still often seemed inexact, and for a while the problem to (more) efficiently estimate Thurston-Bennequin invariants remained open. The first apparently significant progress was achieved in [Kan98], where, on a very special class, an estimate was found improving that of Bennequin's inequality, thereby in particular solving Bennequin's problem about the maximal Thurston-Bennequin invariant of the negative trefoil.

Later, mainly by the work of Fuchs and Tabachnikov [FT97] (and some subsequent papers, see e.g. [CGM00]), the link polynomials were introduced to the subject. They were then applied on specific classes of links to give Thurston-Bennequin invariant estimates [DL01, DM01]. However, the classes considered there (connected sums of two $(2, \cdot)$-torus links and $n$-trivial links of braid index $\leqslant n$ ) are rather narrow, and the proofs do not make use of a deeper study of the polynomials of the links in question. Thus one is interested in general results on Thurston-Bennequin invariants via more intrinsic properties of link polynomials. One approach to such a type of results was given in [Stoe], building on estimates of the coefficients of the polynomials and the description of canonical Seifert surfaces studied previously in [Sto01]. It was remarked that several classes of knots and links can be made to fit into the picture, although the argument of [Stoe] does not always suffice, and additional work is necessary.

In this paper we will carry out this work on the class of mirrored almost positive knots. The additional ingredient needed comes from an originally quite unrelated direction, namely that of Gauß diagram formula invariants [PV94, Fie01]. These formulae have several related knot theoretic applications, which we also discuss. The reason why we consider almost positive knots is because they form the most general class of knots to which the Gauß diagram methods appear applicable

Received 24 May 2002, accepted in final form 18 September 2002.

2000 Mathematics Subject Classification 57M25 (primary), 57N10, 53D10, 57M15 (secondary).

Keywords: almost positive knot, unknotting number, genus, link polynomial, signature, achiral.

This journal is (c) Foundation Compositio Mathematica 2004. 
with reasonable effort. We thus apply the Gauß diagram formulae to incorporate the ThurstonBennequin invariant inequalities for almost positive knots into a larger knot theoretic setting.

To state our results, we first make some definitions.

Many properties of knots are defined by the existence of diagrams with certain properties. Such classical properties are alternation and positivity. By adjoining the word 'almost' before the name of the property, we mean that the knot does not have a diagram with that property, but one in which it can be attained by one crossing change. Here we consider the notion for positivity.

Definition 1.1. The writhe is a number $( \pm 1)$, assigned to any crossing in a link diagram. A crossing as in Figure 1(a) has writhe 1 and is called positive. A crossing as in Figure 1(b) has writhe -1 and is called negative.

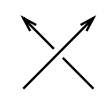

(a)

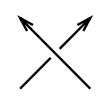

(b)

Figure 1. A positive $(a)$ and a negative $(b)$ crossing.

Definition 1.2. A knot is called positive if it has a positive diagram, i.e. a diagram with all crossings positive. A knot is called almost positive if it is not positive, but has an almost positive diagram, i.e. a diagram with all crossings positive except one.

Recently, the Fiedler-Polyak-Viro approach [PV94, Fie01] to Vassiliev invariants [Bar95, Bar, Vas90] via Gauß diagram formulae gave a new powerful tool in studying positivity, see [Stoa].

The aim of the present paper is to extend the applications of the Fiedler Gauß diagram sum formula to almost positivity. In particular we will apply this formula to classify almost positive diagrams of the unknot (Corollary 3.2). More generally, we will show that knots with zero or negative Fiedler invariant cannot be almost positive. As achiral knots have zero Fiedler invariant, this means in particular that any almost positive knot is chiral.

We also show chirality for the untwisted Whitehead doubles of an almost positive knot (with either clasps), which follows from the positivity of its Casson invariant $v_{2}$ ( $\S 4$ ). As a further consequence we prove that an almost positive knot itself has non-trivial polynomial invariants. This is proved for the Jones polynomial (and hence also for the HOMFLY, or skein, [FHL85] and Kauffman [Kau90] $F$ polynomial), but also for the Alexander polynomial [Ale28] and the $Q$ polynomial of Brandt-Lickorish-Millett [BLM86] and Ho [Ho85].

In $\S 5$ we improve the positivity result for $v_{2}$ on almost positive knots of given genus to an estimate involving the crossing number, similar to the one on positive knots in [Stoa]. This allows us to extend a result of [Sto01] to almost positive knots.

TheOREm 1.1. The number of almost positive knots of given genus grows polynomially in the crossing number. That is,

$$
\#\{K: K \text { almost positive knot, } c(K)=n, g(K)=g\}=O\left(n^{p_{g}}\right),
$$

for some number $p_{g} \in \mathbb{N}$, where $O$ denotes the asymptotic behaviour as $n \rightarrow \infty$. The same statement holds if we replace the genus $g(K)$ by the unknotting number $u(K)$ (i.e. consider the number of knots of a given unknotting number).

The positivity result for $v_{2}$ has a consequence for the behaviour of the degrees of the skein polynomial of almost positive knots. An application of this is the proof that Thurston-Bennequin invariants of Legendrian mirrored positive and almost positive knots become arbitrarily small. 


\section{A. Stoimenow}

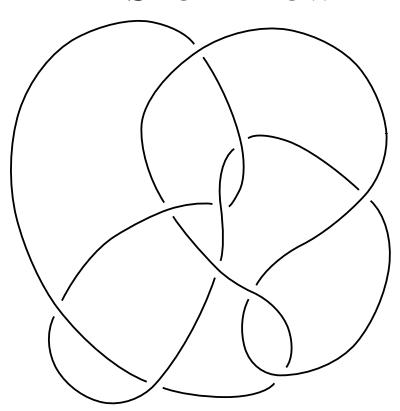

FIGURE 2. The knot $! 10_{145}$ is almost positive.

(Here, and in the sequel, for a knot $K, ! K$ denotes the obverse, or mirror image, of $K$. The definition of $t b$ and $\mu$ is recalled in $\S 5$.)

Theorem 1.2. Let $\left\{K_{i}\right\}$ be distinct positive or almost positive knots and $\left\{\mathcal{K}_{i}\right\}$ be Legendrian embeddings of $! K_{i}$ in the standard contact space $\left(\mathbb{R}^{3}(x, y, z), d x+y d z\right)$ with Thurston-Bennequin invariants $t b\left(\mathcal{K}_{i}\right)$ and Maslov (rotation) numbers $\mu\left(\mathcal{K}_{i}\right)$. Then

$$
t b\left(\mathcal{K}_{i}\right)+\left|\mu\left(\mathcal{K}_{i}\right)\right| \underset{i \rightarrow \infty}{\longrightarrow}-\infty .
$$

The same statement holds for $\left\{\mathcal{K}_{i}\right\}$ transverse, when omitting the $\mu\left(\mathcal{K}_{i}\right)$ term.

This result, which followed for positive knots already from [Stod], extends the aforementioned first series of such examples constructed by Kanda [Kan98], and thus generalizes his result qualitatively. (How to obtain an explicit estimate in terms of genus and crossing number will be discussed later; see the end of $\S 5$.)

Finally, in $\S 6$ we give a proof that almost positive knots have positive signatures.

\section{Gauß diagram sums}

The 'almost' concept was introduced by Adams et al. [Ada92] for alternation. Almost alternating knots are much more common than almost positive, but their diversity makes proofs of specific properties difficult.

The simplest example of an almost positive knot is given in Figure 2.

Example 2.1. The knot $! 10_{145}$ of [Rol76] is almost positive, as its diagram in Figure 2 shows, but it is known not to be positive [Cro89].

We use the Alexander-Briggs notation and the Rolfsen [Rol76] tables to distinguish between a knot and its obverse. 'Projection' is the same as 'diagram', and this means a knot or link diagram. Diagrams are always assumed to be oriented.

So far much less seems to be known about almost positive knots than about almost alternating knots. However, the concept of Gauß diagram sum invariants developed by Fiedler et al. [Fie01, PV94] has several direct applications to such knots. We recall the basic parts of this concept now.

Definition 2.1 [Fie01]. A Gauß diagram of a knot diagram is an oriented circle with arrows connecting points on it mapped to a crossing and oriented from the preimage of the undercrossing to the preimage of the overcrossing.

Example 2.2. As an example, Figure 3 shows the knot $62_{2}$ in its commonly known projection and the corresponding Gauß diagram. 


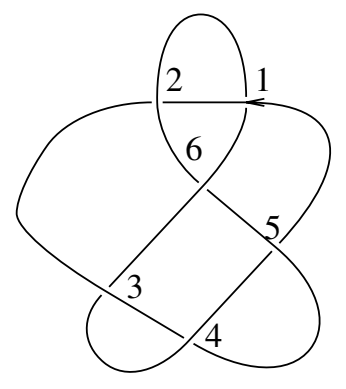

3

Figure 3. The standard diagram of the knot $6_{2}$ and its Gauß diagram.

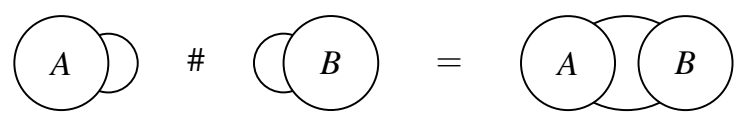

Figure 4. Connected sum of diagrams.

Fiedler [Fie01, FS00] found the following formula for (a variation of) the degree-three-Vassiliev invariant using Gauß diagram sums:

$$
v_{3}=\sum_{(3,3)} w_{p} w_{q} w_{r}+\sum_{(4,2) 0} w_{p} w_{q} w_{r}+\frac{1}{2} \sum_{p, q \text { linked }}\left(w_{p}+w_{q}\right),
$$

where the configurations are

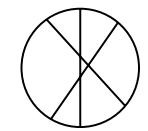

$(3,3)$

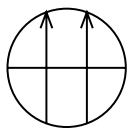

$(4,2) 0$

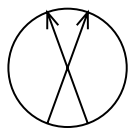

$p, q$ linked

Here the chords depict arrows which may point in both directions and $w_{p}$ denotes the writhe of the crossing $p$. For a given configuration, the summation in (1) is done over each unordered pair/triple of crossings, whose arrows in the Gauß diagram form that configuration. If two chords intersect, we call the corresponding crossings linked. If $p$ and $q$ are linked, and $p$ 's over-crossing is followed by the under-crossing of $q$ when passing the diagram in the orientation direction, we call $p$ distinguished. We will denote by $l k(D)$ the number of linked pairs in a diagram $D$.

To make precise which variation of the degree-3-Vassiliev invariant we mean, we noted in [FS00], that

$$
v_{3}=-\frac{1}{3} V^{(2)}(1)-\frac{1}{9} V^{(3)}(1),
$$

where $V$ is the Jones polynomial [Jon85]. We noted also that $v_{3}$ is asymmetric, i.e. $v_{3}(! K)=-v_{3}(K)$, so that achiral knots have zero invariant.

Definition 2.2. The diagram on the right-hand side of Figure 4 is called the connected sum $A \# B$ of the diagrams $A$ and $B$. If a diagram $D$ can be represented as the connected sum of diagrams $A$ and $B$, such that both $A$ and $B$ have at least one crossing, then $D$ is called disconnected (or composite), else it is called connected (or prime).

Definition 2.3. A crossing $p$ in a knot diagram $D$ is called reducible (or nugatory) if it is linked with no other crossing. Then $D$ looks like the left-hand side of Figure 5. $D$ is called reducible if it has a reducible crossing, else it is called reduced. The reducing of the reducible crossing $p$ is the 


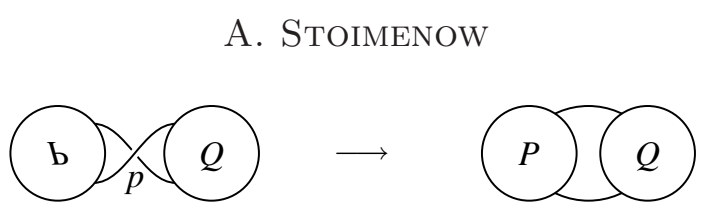

FiguRE 5. Removing nugatory crossings.

move depicted on Figure 5. Each diagram $D$ can be (made) reduced by a finite number of these moves.

Definition 2.4. For two chords in a Gauß diagram $a \cap b$ means ' $a$ intersects $b$ ' (or crossings $a$ and $b$ are linked) and $a \nsupseteq \backslash b$ means ' $a$ does not intersect $b$ ' (or crossings $a$ and $b$ are not linked).

In [Stoa], we gave the following two properties of Gauß diagrams, which we will use extensively in the following.

Lemma 2.1 (Double connectivity $2 C(a, b, c)$, [Stoa]). Whenever in a Gauß diagram $a \cap c$ and $b \cap c$ then either $a \cap b$ or there is an arrow $d$ with $d \cap a$ and $d \cap b$.

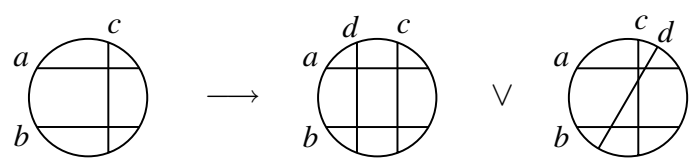

Lemma 2.2 (Even valence $e v(c)$, [Stoa]). Any chord $c$ in a Gauß diagram has odd length, i.e. an even number of basepoints on both its sides or, equivalently, an even number of intersections with other chords.

The first step is to show the following theorem, implying that knots with negative Fiedler invariant are not almost positive.

THEOREM 2.1. In any almost positive diagram $K$ we have $v_{3}(K) \geqslant 0$.

Proof. The idea is to show that for any negative configuration in the Gauß diagram sum, that is, a configuration with a negative weight, we can find a positive configuration, that is, a configuration with a positive weight, which 'equilibrates' it. Such positive configurations we will call accordingly 'equilibrating'.

Let $p$ be the arrow in the Gauß diagram corresponding to the negative crossing. There are three types of negative configurations as follows.

i)

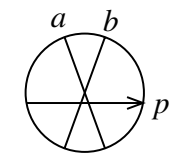

This is equilibrated by $(a, b)$ linked.

ii)

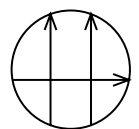

By $2 C(a, b, p), \exists c: c \cap a, c \cap b$ :

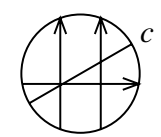

Then $(a, b, p)$ is equilibrated by $(a, b, c) \in(4,2) 0$.

iii)

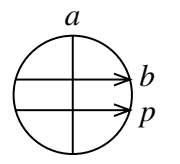

This is equilibrated by $(a, b)$ linked.

It remains to note that no positive configuration equilibrates this way more than one negative configuration. 


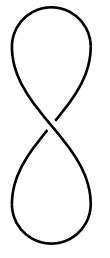

(a)

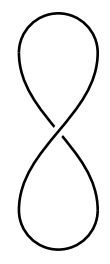

(b)

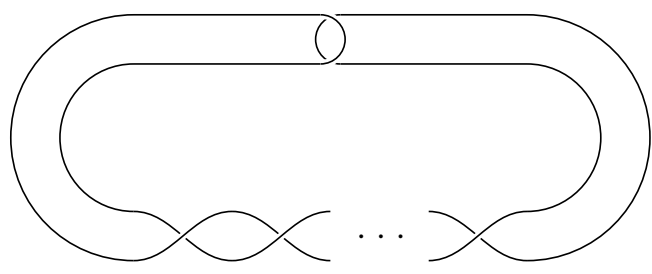

(c)

Figure 6. The possible prime factors of almost positive unknot diagrams.

To extend the result, we like to show that, except in the desired cases, non-equilibrating positive configurations exist, and therefore the value of the Gauß diagram sum is positive. To do so, we will study the 'environment' of the negative arrow $p$ in the Gauß diagram. In most cases we will make assumptions, then by $2 C$ and $e v$ we will show the existence of further and further arrows in the Gauß diagram, leading at some point unavoidably to the creation of a non-equilibrating configuration. Therefore these assumptions turn out wrong and leave only the desired cases. In the following section we explain the tricky details of this obvious idea.

\section{The classification of almost positive unknot diagrams}

Playing a central role in knot theory, researchers have been trying for a long time to identify the unknot from its diagrams and to classify them. This was achieved in theory by an algorithm of Haken [Hak61]. This algorithm, unfortunately, but not unexpectedly, is far too complex to give (or even let us hope for) some nice explicit description of all diagrams of the unknot. (See also [HL01].) Other attempts have been made using braid representations. In this case, the question of which conjugacy classes of 4-braids have unknotted closure is already known to be extremely hard [Mor86, Fie93].

More is known for special cases of diagrams. It has been proved via different methods that alternating [Cro89, Mur87, Gab86] or positive [Cro89, Stoa] diagrams of the unknot are completely reducible, that is, transformable into the zero crossing diagram by (crossing number) reducing Reidemeister I moves only. (One common argument is that in such diagrams the Seifert algorithm must give a disc, and these are exactly the diagrams with this property.)

For almost positivity, the following appealing series of examples comes to mind: the twist knots $3_{1}, 4_{1}, 5_{2}, 6{ }_{1}, 7_{2}, 8_{1}, \ldots$, that is, the knots with Conway notation $(k, 2), k \in \mathbb{N}$, can be unknotted in their alternating diagrams by one crossing change, giving (modulo mirroring) an almost positive diagram, see Figure 6(c).

Here we will show that for connected diagrams these are indeed the only examples, which leads to a classification of all almost positive diagrams of the unknot.

Note, that this result again gives a strong contrast to the problems of controlling almost alternating diagrams of the unknot [Ada94, $\S 5.5]$.

TheOREm 3.1. If $K$ is a connected almost positive diagram and $v_{3}(K)=0$, then $K$ is an unknotted twist knot diagram (see Figure 6(c)) or a one crossing diagram.

Our proof is based on some very involved analysis of the combinatorics of the Fiedler formula, similar to that of [Stoa], and is divided into several subcases. To introduce some abbreviations, in the following ' $\not$ ' denotes a contradiction and ' $\|$ ' denotes 'parallel' (see Figure 7). It appears more appropriate to use a rather symbolic notation (even if it reduces readability), to avoid misinterpretations of the wording, as some logical constructs that will appear will be rather complex. 


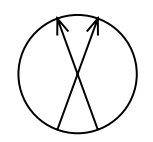

$p \cap q$
A. Stoimenow

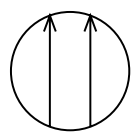

$p \| q$

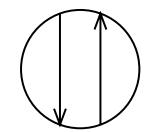

$p \nmid q$

Figure 7. The possible mutual positions of two arrows in a Gauß diagram.

Proof. Fix $K$ and its negative crossing $p$. $K$ has no non-equilibrating positive configurations. Therefore the following conditions hold in $K$ (in the following we will refer to each one by boxing its number):

1) If $r, q$ are linked, $p \notin\{r, q\}$, then at least one of $r, q$ is linked with $p$. If not both are linked with $p$, then the not linked one is $\| p$.

2) $p$ is in any $(3,3)$ configuration. That is, whenever $a, b$ and $c$ form a $(3,3)$ configuration, $p$ is one of $a, b$ or $c$.

3) If $a \| b, p \notin\{a, b\}$ and $\exists c \neq p$ with $(a, b, c) \in(4,2) 0$, then $c$ is unique with this property and $(a, b, p) \in(4,2) 0$.

4) A fragment of the kind

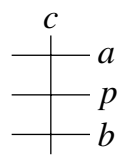

with $c \cap a, b, p$ and $a \not \supset b \not x \not \backslash a$ does not exist. Else $a \| p$ (else $(c, a) \in[$ ) and $b \| p$ (else $(c, b) \in 1$ ), so $a \| b$ and $(a, b, c) \in(4,2) 0$ 々 to 3 .

5) A fragment of the kind

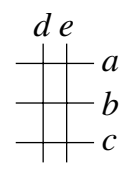

with $p \notin\{a, b, c, d, e\}$ and $e, d \cap a, b, c$ does not exist. Else $a \cap b$ or $b \cap c$ leads to a $(3,3)$-configuration ( $\{$ to 2 ) and for $a \not \chi b$ and $b \not \chi c$, two of $\{a, b, c\}$ are $\|$, say $(a, c)$. Then $(a, c)$ participate in at least two $(4,2) 0$ configurations, $\&$ to 3 .

Case 1. $\exists a, b \cap p, a \cap b$.

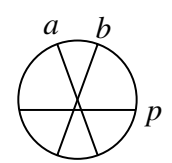

Assume

$$
\exists c: c \nsupseteq a, b, c \cap p
$$

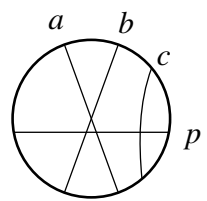

Then $2 C(a, c, p) \Rightarrow \exists d: d \cap a, d \cap c$ and $2 C(b, c, p) \Rightarrow \exists d^{\prime}: d^{\prime} \cap b, d^{\prime} \cap c$. Now if $d \cap a, b$, then 
$(d, a, b) \in(3,3) \xi$, and if $d^{\prime} \cap a, b$, then $\left(d^{\prime}, a, b\right) \in(3,3) \xi$, so $d^{\prime} \not \not a, d \not h b$ and in particular $d \neq d^{\prime}$.

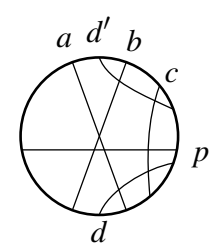

Furthermore, $d \not d^{\prime}$ (if $d \cap d^{\prime}$, then by $d, d^{\prime} \cap c$ we had $\left(d, d^{\prime}, c\right) \in(3,3) \xi$ ), and therefore (see diagram) not both $d, d^{\prime} \cap p$. On the other hand, $d, d^{\prime} \not x p$ implies $\left(c, d, p, d^{\prime}\right) \in 4$ 々, so $p$ is intersected by exactly one of $d, d^{\prime}$. Assume w.l.o.g. (modulo mirroring the diagram) that $d \cap p, d^{\prime} \rtimes p$.

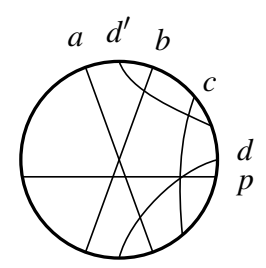

As a result of $2 C\left(d, c, d^{\prime}\right)$ we have $\exists e \cap d, d^{\prime}$. We have $e \cap p$ (else $\left(e, d^{\prime}\right) \in 1$ ) and $e \not X a, b, c$ (else 2 ). There are two possibilities for $e$ as follows:
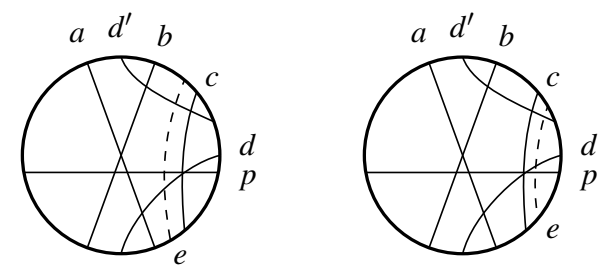

Both choices are equivalent (the second is the same as the first with $c, e$ swapped), so we consider only the first, i.e.

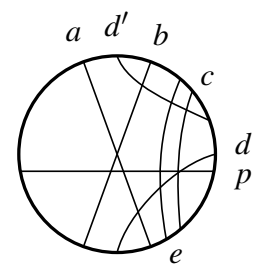

Now $2 C\left(d^{\prime}, b, a\right) \Rightarrow \exists g: g \cap a, g \cap d^{\prime}$. Then $g \not d, e, c, b$ (else 2 and $g \cap p$ (else $\left(g, d^{\prime}\right) \in[$ ). So (modulo swapping $b$ and $g$ ) we obtain the following diagram.

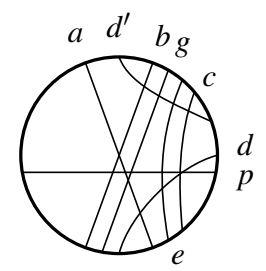

Now $e v(b) \Rightarrow \exists h: h \cap b$. Then $h \not \backslash g\left(\right.$ else $\left(b, g, h, a, d^{\prime}\right) \in 5$ ) and $h \not \backslash a, d^{\prime}$ (else 2 ). There are three 


\section{A. Stoimenow}

choices of $h$ indicated by dashed arcs in the following diagram.

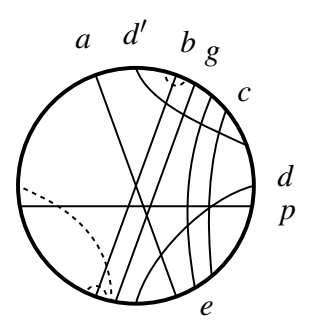

If $h$ is one of the chords in the lower part of the diagram, then apply $2 C\left(h, b, d^{\prime}\right)$. So $\exists i: i \cap h, i \cap d^{\prime}$. But then $i \cap a$ and $\left(a, d^{\prime}, b, i, g\right) \in 5$ z. If $h$ is the chord in the upper part of the diagram, then apply $2 C(h, b, a)$ to deduce $\exists i: i \cap h, i \cap a$. But then $i \cap d^{\prime}$ with the same contradiction.

This shows, that the assumption (3) is wrong, and, using the fact that $c \neq a, b$ with $c \cap a, b$ leads to 2 , we have proved

$$
\forall c \neq a, b: c \cap p \Rightarrow(c \cap a \wedge c \not \backslash b) \vee(c \cap b \wedge c \not x a) .
$$

Now assume

$$
\exists c: c \cap b, c \nsupseteq x a, c \nsupseteq p
$$

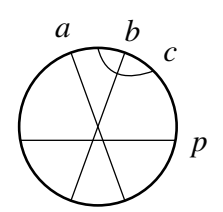

The case $\exists c: c \cap a, c \nsupseteq \not b, c \nsupseteq p$ is dealt with analogously. Now $2 C(a, c, b) \Rightarrow \exists d: d \cap c, a . d \not \supset b$ (else 2 ) and $d \cap p$ (else $(d, c) \in 1$ ). Modulo swapping $b$ and $d$ one obtains a diagram as follows:

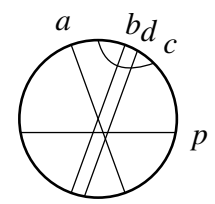

Now $e v(d) \Rightarrow \exists e: e \cap d$. Then $e \not \supset b$ (else $(b, d, c, a, e) \in 5$ ) and $e \not \subset c, a$ (else 2 ). There are two choices of $e$ :
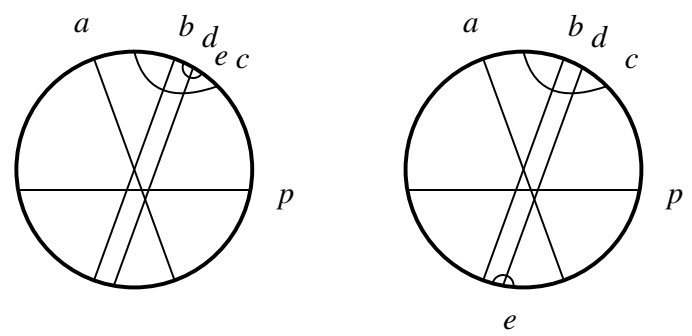

In the first case apply $2 C(e, d, a)$ to deduce $\exists f: f \cap e, a$. But then $f \cap c$ and $(a, c, b, d, f) \in 5$ 々. In the second case apply $2 C(e, d, c)$. Then $\exists f: f \cap e, c$. But then $f \cap a$ with the same contradiction.

This shows a contradiction to assumption (5), so that we obtain $(c \cap a \vee c \cap b) \Rightarrow c \cap p$, and together with (4) we have

$$
\forall c \neq a, b:(c \cap a \vee c \cap b) \Longleftrightarrow c \cap p
$$

Assume

$$
\begin{aligned}
\exists c: c \cap p, & c \cap a, \quad c \nsupseteq b \\
\wedge \exists c^{\prime}: c^{\prime} \cap p, & c^{\prime} \nsupseteq a, c^{\prime} \cap b .
\end{aligned}
$$




\section{GAuss DiAgram SUmS ON ALmost POSITIVE KNOTS}

Case 1.1. $c \cap c^{\prime}$. Modulo swapping $(b, c)$ and $\left(a, c^{\prime}\right)$ we obtain the following:

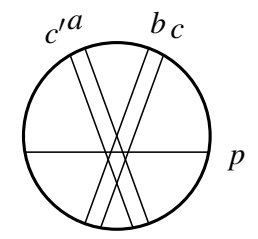

Now $e v(a) \Rightarrow \exists d: d \cap a$. Then $d \not \bigcap b, c$ (else a (3,3)-configuration of 2 ) and $d \not \supset c^{\prime}$ (else $\left(c^{\prime}, a, b, c, d\right)$ $\in 5)$. There are three choices for $d$ :
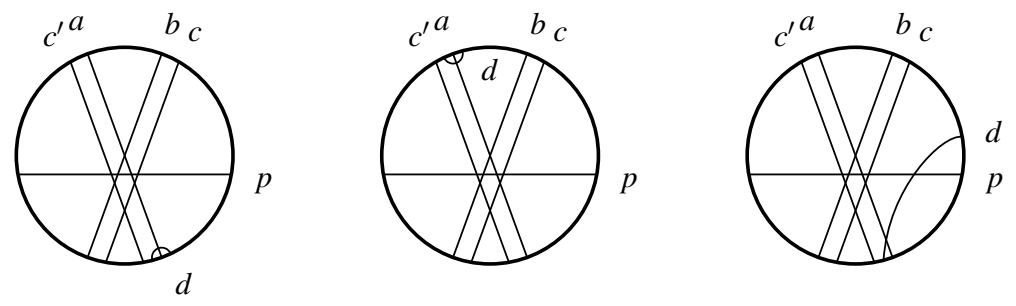

In the first case use $2 C(d, a, b)$. So $\exists e: e \cap d, e \cap b$. Then $e \cap c$ and $\left(b, c, a, e, c^{\prime}\right) \in 5$ z. In the second case use $2 C(d, a, c)$, so that $\exists e: e \cap d, c$. Then $e \cap b$ with the same contradiction. In the third case use the symmetry of the diagram to obtain a $d^{\prime}$ like

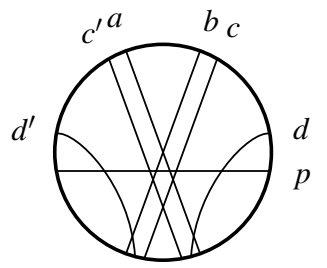

(or apply one of the other two cases to $d^{\prime}$ to obtain a contradiction). Then $2 C\left(d, d^{\prime}, p\right) \Rightarrow \exists e$ : $e \cap d, e \cap d^{\prime}$. But then $e \cap c, e \cap c^{\prime}$, which together with $c \cap c^{\prime}$ implies $\left(c, c^{\prime}, e\right) \in(3,3)$ i to 2 .

Case 1.2. $c 內 c^{\prime}$.

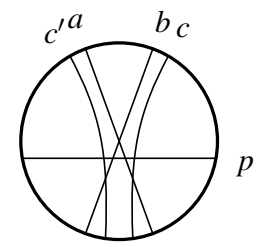

As a result of $2 C\left(c^{\prime}, p, c\right)$ we have $\exists d: d \cap c, c^{\prime}$. Then $d \not x a, b$ (else 2 ). So the diagram looks like

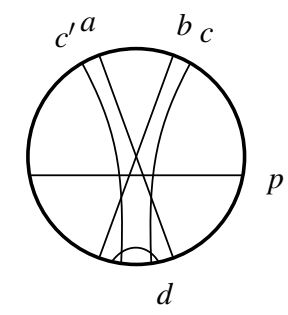

Now $2 C\left(d, c^{\prime}, b\right) \Rightarrow \exists e: e \cap d, e \cap b$. Then $e \cap p$ (else $(e, d) \in\left[1\right.$ ) and $e \not \subset c, c^{\prime}, a$ (else 2 ). In the same way, $2 C(d, c, a) \Rightarrow \exists f: f \cap a, d$, and $f \cap p, f \not \subset c, c^{\prime}, b$. Furthermore, $f \not \subset e$ (else $\left.(f, e, d) \in(3,3)\right)$. 


\section{A. Stoimenow}

Modulo swapping $(f, c)$ and $\left(e, c^{\prime}\right)$ we obtain

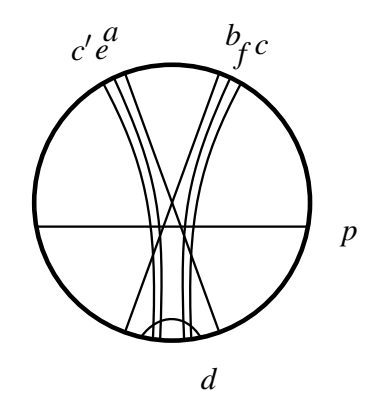

Now $e v(e) \Rightarrow \exists g: g \cap e$. For this $g, g \nsupseteq d, b$ (else 2 $), g \nsupseteq c^{\prime}$ (else $\left(c^{\prime}, e, b, d, g\right) \in 5$ ) and $g \cap p$ (else $(g, p, d, e) \in 4$ ). However, a glimpse at the above diagram shows that no such $g$ exists. Therefore, this contradiction shows that our assumption (7) is wrong, and we have

$$
\exists i \in\{a, b\}: \forall c \neq a, b: p \cap c \Longleftrightarrow p \cap i \wedge p \not \bar{i}
$$

with $\bar{i} \in\{a, b\} \backslash\{i\}$. Assume w.l.o.g. that $i=a$. As a consequence of (8), all $c \neq a: p \cap c$ do not mutually intersect (else an intersecting pair would build a $(3,3)$-configuration with $a$ ). Then the subdiagram made up of $p$ and all its neighbours, i.e. all crossings linked with $p$, looks like

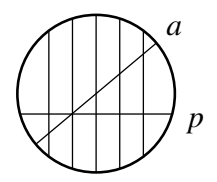

Assume now that there are more chords in the Gauß diagram. So by connectedness $\exists d: d \cap c$ for some $c \cap p$ and $d \rtimes p$; in particular, $c \neq a($ as $d \cap a \Rightarrow d \cap p)$.

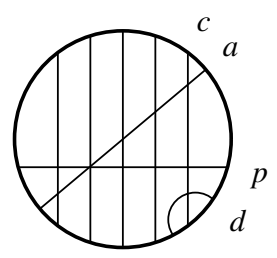

But then $e v(c) \Rightarrow \exists d^{\prime} \cap c \cdot d^{\prime} \backslash \backslash d$ (else 2) and by assumption $d, d^{\prime} \not x$, so $\left(c, d, p, d^{\prime}\right) \in 4$ 々. Therefore there are no more chords in the Gauß diagram than those of (9), and the knot diagram is an unknotted odd crossing number twist knot diagram.

Case 2. No pair $a, b$ with $a, b \cap p$ is linked. Then the subdiagram of the Gauß diagram made up of $p$ and all crossings linked with it looks like

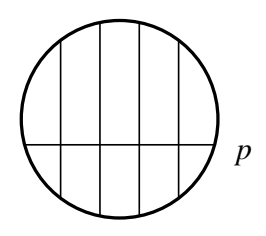

By the assumption and exclusion of 2 and 4 , no chord intersecting $p$ is intersected more than once else. By even valence, then any $c: c \cap p$ must be intersected exactly once else.

Now assume $a, b \cap p$. Then by $2 C(a, b, p)$ the second chord intersecting $a$ and $b$ must be the same for all $a, b \cap p$. 
But then the Gauß diagram looks like

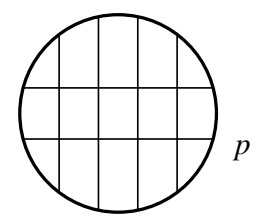

and the knot diagram is an unknotted even crossing number twist knot diagram.

Two immediate consequences of the theorem are as follows.

Corollary 3.1. No knots with $v_{3}=0$ (inter alia, no achiral knots) can be almost positive.

Corollary 3.2. All almost positive unknot diagrams are connected sums of diagrams as in Figure 6 with summands of the kind (b) and (c) appearing together exactly once.

These results seem to have been first obtained (without published proof) by Przytycki and Taniyama [PT91], and have been recently recovered in a nice way by Rudolph [Rud99]. A special case of Corollary 3.2 (for almost alternating almost positive diagrams) was independently proved by Hirasawa [Hir00].

The referee also informed me of a related (unpublished) result of Tat Sang Fung. He showed that almost ascending (descending) knots, i.e. knots with diagrams ascending (descending) except at one crossing, are twist knots.

\section{Whitehead doubles and the Casson invariant}

The use of Vassiliev invariants allows us to extend the previous chirality results to certain cables of positive and almost positive knots.

DeFinition 4.1. In the following diagram we summarize three kinds of clasps in a knot diagram and how we will refer to them (the diagrams are understood up to mirroring and strand orientation, when the latter is not specified).

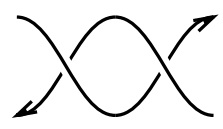

reverse clasp

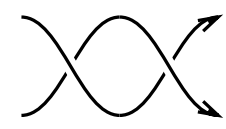

parallel clasp resolved clasp

Formally, a clasp is a digon, a connected component of the complement of the diagram, neighbouring just two crossings. It can be identified with the (unordered) pair of these two crossings. (A pair cannot form several clasps in a knot diagram.)

The idea to consider untwisted Whitehead doubles came out of a nice relationship between the Vassiliev invariants of degrees two and three. Let $v_{2}$ denote the Vassiliev invariant of degree two given by

$$
v_{2}=-\frac{1}{6} V^{\prime \prime}(1)=[\nabla(z)]_{z^{2}}
$$

(in the following $\Delta$ is the Alexander polynomial, $\nabla$ the Conway polynomial [Con69] and $P$ the HOMFLY polynomial) and $w_{ \pm}$denotes the untwisted double with positive (respectively negative) clasp. Then in [Stoa] it was proved that

$$
v_{3}\left(w_{ \pm}(K)\right)= \pm 8 v_{2}(K) .
$$




\section{A. Stoimenow}

This follows basically from a combination of two observations of McDaniel and Rong [MR01] (that the dualization of a double operation of a knot maps Vassiliev invariants to Vassiliev invariants) and Lin [Lin94] (that this endomorphism is nilpotent).

Whitehead doubles are classical examples of knots with $\Delta=1$ and so have been under some consideration in connection with the often raised question about a non-trivial knot $K$ with $V_{K}=1$ (or even $P_{K}=1$ ). Lickorish and Thistlethwaite [LT88] excluded Whitehead doubles of adequate knots from having this property, and in [Rud90], as quoted in [KL98], it was shown that for $K$ positive, $P\left(w_{ \pm}(K)\right) \neq 1$. The latter result inspired the strengthening of it in [Stoa] by combining (10) with the lower bound for $v_{2}$ in positive diagrams in [Stoa], showing that in fact for $K$ positive, $w_{ \pm}(K)$ has a non-zero degree-three-Vassiliev invariant, and hence in particular is chiral and has a non-trivial Jones polynomial. The extension of this result to almost positive knots (Corollary 4.2) then follows from an inequality for $v_{2}$ (stated in Theorem 4.1 below) similar to the condition on $v_{3}$ in Theorem 3.1.

Note that for Whitehead doubles neither the signature, nor the Bennequin inequality work (in some easy way) to show chirality. However, the referee informed us that in a recent paper [JLWW02], Jiang et al. (using more advanced techniques) showed that a (not necessarily untwisted) Whitehead double is achiral if and only if it is the unknot or the figure-8-knot.

Unfortunately, the need to exclude the knotted cases forces us to reprove, this time using $v_{2}$, the result on almost positive unknot diagrams. Fortunately, this time the proof is somewhat easier.

Theorem 4.1. If $D$ is a connected almost positive diagram then $v_{2}(D) \geqslant 0$, and if $v_{2}(D)=0$, then $D$ is an unknotted twist knot diagram or a one crossing diagram.

An immediate corollary of this theorem generalizes a recent result of Menasco and Zhang [MZ00] on the property $P$ conjecture [BM71] (see e.g. [BS88, DR99, CG88]).

Corollary 4.1. Almost positive knots have property $P$ (i.e. every non-trivial surgery on $S^{3}$ along such knots produces a non-simply connected manifold).

Proof. This follows from the surgery formula for the Casson invariant of homology spheres [AM90] (see also $[\mathrm{MZ00}, \S 1]$ ).

For the proof of Theorem 4.1 we need some preparations. In addition to $e v$ and $2 C$ we will need the following elementary observation.

Lemma 4.1 (Extended even valence eev(c), see [Stoa]). In the Gauß diagram of a positive knot diagram, exactly one half of the arrows intersecting any chord $c$ intersect it in one or the other direction (that is, are distinguished or not in the resulting linked pair).

We will need the following Gauß diagram sum formula for $v_{2}$ which is due to Polyak and Viro [PV94, (4) and p. 451]:

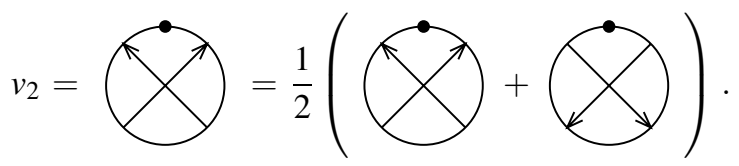

The point on the circle depicts a point to be put somewhere on the knot curve in the diagram, but not at a crossing. 
Proof of Theorem 4.1. The fact that $v_{2}(D) \geqslant 0$ is almost positive diagrams may be proved along similar lines as $v_{3}$, but it is easier to quote Cromwell's (skein-theoretic) proof [Cro89, Corollary 2.2, p. 539].

Now consider an almost positive knot diagram $D$ and its Gauß diagram with the negative arrow $k$. Recall the move we used in the proof of the positivity of $v_{2}$ in positive diagrams in [Stoa]:
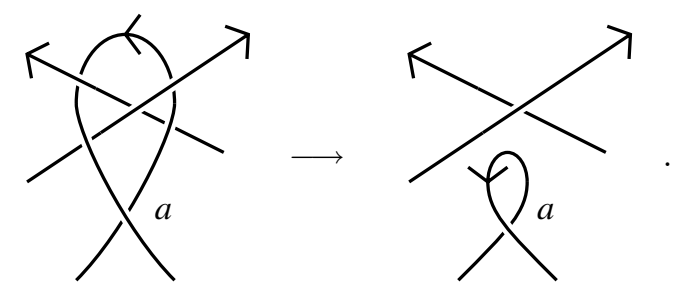

We trivialized loops by pulling them above the rest of the diagram by crossing changes. On the level of Gauß diagrams this means that we delete a chord $a$, intersecting all chords ending on one of its sides, and then also delete all these intersecting chords.

For the following arguments it is convenient to place the point of (11) near one of the endpoints of $a$. Now consider what happens with $v_{2}$ under our move.

It is clear that if $a \nsupseteq k$, then the move never augments $v_{2}$, because the only negative (contribution) configurations removed are those of $(k, c)$ with $c \cap a$, but their contribution is equilibrated by those of $(c, a)$. Moreover, because of $e v$ and $e e v$, an even number of $c$ with $c \cap a$ intersects $k$ (because after the move the Gauß diagram still corresponds to a knot diagram), an even number of them does not intersect $k$, and exactly half of these numbers intersect $a$ in either direction. Hence if $a \cap c$ with $c \not h k$, resolving $a$ would strictly reduce $v_{2}$ (as $a$ is linked with some $c$ by connectedness) and leave over an almost positive diagram. Therefore, after the move still $v_{2} \geqslant 0$, and hence before the move $v_{2}>0$. So, for any $a, a \cap k$ or $a$ intersects all $c \cap k$. This splits the positive arrows into two parts

$$
\underbrace{\{c: c \cap k\}}_{A} \cup \underbrace{\{c: c \not \backslash k \wedge \forall d \cap k: c \cap d\}}_{B}
$$

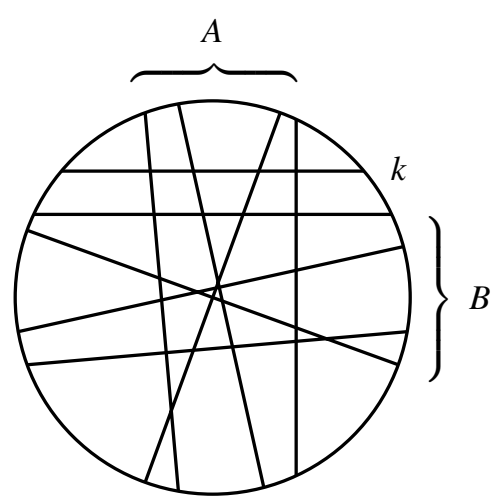

Now putting the point on some segment of the circle between an endpoint of a chord in $A$ and an endpoint of a chord in $B$ and using $e v$ and $e e v$ we see that any $c \in B$ gives a positive contribution to $v_{2}$ equilibrating the negative contribution of $k$. Hence for $v_{2}=0$ we must have $|B| \leqslant 1$.

If $B=\{c\}$, then $c$ forms with $k$ an (unlinked) resolved clasp, whose elimination by a Reidemeister II move gives a positive diagram with $v_{2}=0$. This diagram must then have only isolated chords. Hence $A$ has only non-mutually intersecting arrows (or non-linked crossings in the 


\section{A. Stoimenow}

knot diagram), whose number by $e v$ must be even, so we have

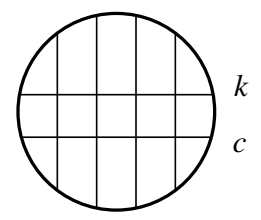

an unknotted twist knot diagram with an even crossing number.

If $B=\varnothing$, then the knot diagram can be obtained just by Reidemeister II moves and crossing changes from

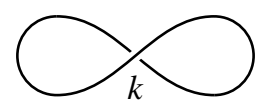

But then clearly the diagram will have a clasp somewhere (at least the one created by the last Reidemeister II move). Hence

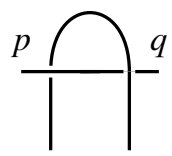

all positive crossings in the diagram can be resolved by consecutively removing the clasps. Putting the point near one of the endpoints of $k$ in the Gauß diagram, one sees because of $e v$ and eev that resolving such a clasp $(p, q)$ never augments $v_{2}$ and that it reduces it strictly if $\exists c \neq k$ with $c \cap p, q$ and $p \cap q$ or if $\exists c_{1}, c_{2} \neq k$ with $c_{1}, c_{2} \cap p, q$ and $p 历 q$.
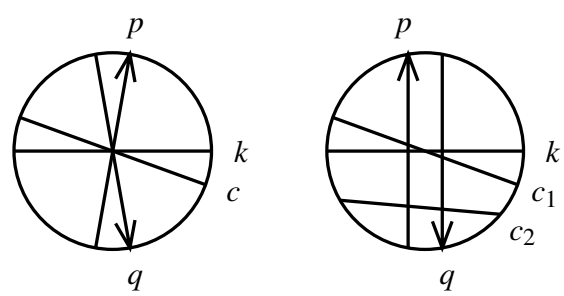

This means that for $v_{2}=0$ no such clasp occurs in the sequence of clasps to be resolved. In particular, there cannot be $a, b, c \cap k$ with $a \cap b, c$ and $b \cap c$

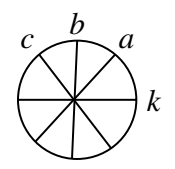

as at some point $a, b$ or $c$ must be involved in a clasp and it would have to be one of the above kinds. Consequently, no subdiagram like

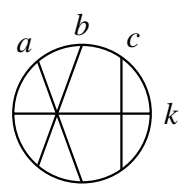

occurs in the Gauß diagram. Else by $2 C(b, k, c), \exists d \cap b, c$. By assumption $d \cap k$, so $d \backslash x a$ (else $d, a, b \in(12))$. But then by $2 C(a, k, c)$ similarly there is some $d^{\prime}$ such that $d, d^{\prime}$ and $c$ 
mutually intersect (contradicting (12)).

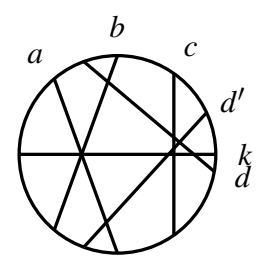

So, if $a, b \cap k$ and $a \cap b$, any $c \cap k$ intersects exactly one of $a$ and $b$. This pairs up the chords intersecting $k$ in two parts of $p$ and $q$ elements, such that $a \cap b$ if and only if $a$ and $b$ belong to different parts. $e v$ forces $p$ and $q$ to be odd

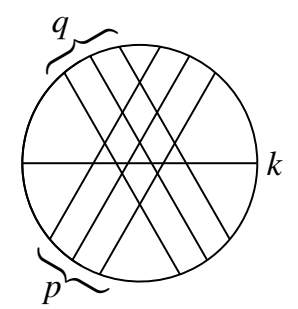

and then the diagram is the diagram of a $(p, q,-1)$ pretzel knot with $p, q>0$ odd. Finally, to show $v_{2}=0$ only if such a knot is unknotted is a matter of direct calculation. It is known (or can be deduced from the formula for $\left.v_{2}\right)$ that $v_{2}(P(p, q, r))=(p q+p r+q r+1) / 4$, so $v_{2}(P(p, q,-1))=0$ implies $p=1$ or $q=1$, and we have an unknotted twist knot diagram of odd crossing number.

COROllary 4.2. The untwisted Whitehead doubles (with either clasps) of an almost positive knot are chiral and have non-trivial Jones polynomial.

Proof. As noted, this is straightforward from Theorem 4.1 and (10).

Clearly the chirality of the satellite follows from that of the companion, hence this result is a consequence of the previous one (Corollary 3.1) also by classical arguments. However, the following corollary shows that the chirality result extends to many cases, where it is less obvious, for example for the connected sum of an almost positive knot and its obverse.

Corollary 4.3. If a knot $K$ is the connected sum of positive, almost positive knots and their obverses, or a cable knot thereof, then its untwisted Whitehead doubles (with either clasps) are chiral, and have non-trivial Jones polynomial.

Proof. If $K$ is a connected sum, then the positivity of $v_{2}$ follows from its invariance under mirroring and additivity under connected sum. As for cables, if $p, q \in \mathbb{N}$ are coprime, $T_{p, q}$ denotes the $(p, q)$-torus knot and $K_{p, q}$ the satellite of $T_{p, q}$ around $K$, we have

$$
v_{2}\left(K_{p, q}\right)=a_{p, q} v_{2}(K)+c_{p, q} .
$$

It is known that the eigenvalues of Vassiliev invariants (modulo Vassiliev invariants of lower degree) under cabling operations are always positive (see [KSA97] or [MR01]; in fact, they are powers of the number of parallels of the cable), hence so is $a_{p, q}$, and putting the unknot in (13) we obtain $c_{p, q}=v_{2}\left(T_{p, q}\right)>0$; hence the positivity of $v_{2}$ for the cable follows from that of its companion.

Finally, we can now exhibit non-triviality of the polynomials for almost positive knots themselves. While for $V$ (and hence for $P$ and $F$ ) we could have concluded non-triviality already in the previous paragraph using $v_{3}$, we postponed it until now in order to do it for all five polynomials at one go. This is another considerable bonus of using Vassiliev invariants instead of signatures as in [PT91]. 


\section{A. Stoimenow}

Corollary 4.4. For any of the polynomials of Alexander-Conway, Jones, HOMFLY, BrandtLickorish-Millett-Ho and Kauffman there is no almost positive knot with unit polynomial.

Proof. Use the positivity of $v_{2}$ and the relations

$$
-6 v_{2}:=-3 \Delta^{\prime \prime}(1)=V^{\prime \prime}(1)=Q^{\prime}(-2)
$$

and the well-known specializations for the HOMFLY and Kauffman polynomial. The equality between the Jones and Alexander polynomial is probably due already to Jones [Jon87, § 12]. The relation between the Jones and Brandt-Lickorish-Millett-Ho polynomial is proved by Kanenobu in [Kan88].

For the Jones (and hence also HOMFLY and Kauffman) polynomial, this result was also announced in [PT91]. An alternative proof, using arguments similar to those of Przytycki and Taniyama, appeared in [Stoc].

\section{Some further inequalities related to the genus}

An obvious desire is to improve the positivity results for $v_{2}$ and $v_{3}$ on almost positive knots to inequalities involving the crossing number, as for positive knots in [Stoa, Theorem 6.1]. Focusing in the following on $v_{2}$, which leads to more interesting consequences, this is related to a conjecture made in [Stoa, $\S 6]$.

Definition 5.1. Call a diagram bireduced if it is reduced, i.e. has no nugatory crossings, and does not admit a move

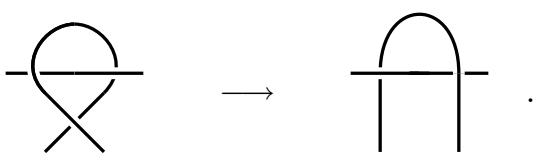

From now on we will assume that all diagrams are reduced.

Conjecture 5.1. In a positive bireduced diagram $D, v_{2} \geqslant l k(D) / 4$, where $l k(D)$ is the number of linked pairs in $D$.

A computer experiment revealed that the conjecture is in general false. One counterexample is a diagram of $8_{19}$ obtained by making the alternating diagram of $9_{40}$ positive by crossing changes. It has 21 linked pairs, but $v_{2}=v_{2}\left(8_{19}\right)=5$.

Nevertheless, a weaker version of the conjecture is true. To formulate the statement, we need to recall the notion of the genus of a diagram. In the following we will also use the weak genus of a knot $K$. Both terms were introduced in [Sto01].

Definition 5.2. For a diagram $D$ of knot $K$, we define the genus $g(D)$ as the genus of the surface obtained by applying the Seifert algorithm to this diagram,

$$
g(D)=\frac{c(D)-s(D)+1}{2},
$$

with $c(D)$ and $s(D)$ being the crossing and Seifert circle number of $D$, respectively. The weak genus of $K$, denoted by $\tilde{g}(K)$, is the minimal genus of all its diagrams:

$$
\tilde{g}(K)=\min \{g(D): D \text { is a diagram of } K\} .
$$

We start with the following technical lemma, which will be of decisive importance later.

Lemma 5.1. For every genus $g$, and every $\varepsilon>0$, there are at most finitely many positive diagrams $D$ of genus $g$ and $l k(D) / v_{2}(D)>4+\varepsilon$. 


\section{GaUss DiAGRAM SUMS ON ALMOST POSITIVE KNOTS}

For the proof, we recall the main result of our work on the diagram genus in [Sto01], which will be used in the following. It is related to the move below which we call a $\bar{t}_{2}$ move:

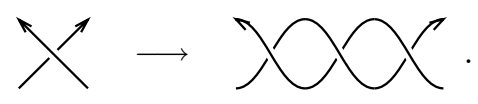

Another move we will use, the flype, is the one of [MT91].

Theorem 5.1 (Theorem 3.1 of [Sto01]). Reduced knot diagrams of given genus decompose into finitely many equivalence classes modulo crossing changes, $\bar{t}_{2}$ moves and their inverses. That is, reduced knot diagrams of given genus with no resolved clasps can all be obtained from finitely many (called 'generating') diagrams by repeated $\bar{t}_{2}$ moves.

A further result we will need later is proved in [Stoa].

Lemma 5.2. [Stoa] If $D$ is a positive bireduced diagram of c crossings, then $l k(D) \geqslant 3\lfloor(c-1) / 2\rfloor$.

Proof of Lemma 5.1. One needs to observe that if $D^{\prime}$ arises from $D$ by a $\bar{t}_{2}$ move, then $l k\left(D^{\prime}\right)-$ $l k(D)=4\left(v_{2}\left(D^{\prime}\right)-v_{2}(D)\right)$. To see this, put the basepoint in (11) near the (crossings of the) created clasp. Then Theorem 5.1 implies that for any sequence $D_{1}, D_{2}, \ldots$ of (distinct) positive diagrams of fixed genus, it holds that $l k\left(D_{i}\right) / v_{2}\left(D_{i}\right) \rightarrow 4$ as $i \rightarrow \infty$.

The number of generating diagrams grows very rapidly with the genus, but in [Sto01, Stob] at least a description of all such diagrams for genus one and two was obtained. This work can be used to make the estimate in Lemma 5.1 more explicit for small genus by accounting for the exceptional cases.

Proposition 5.1. On any positive diagram $D$ of genus at most three, we have $v_{2}(D) / l k(D) \geqslant 5 / 21$.

Proof. To make a systematic verification of the maximal ratio $l k / v_{2}$ on positive diagrams, once having found it to be greater than 4, the argument proving Lemma 5.1 shows that we need to consider just diagrams without reverse clasps. A similar argument also shows the same for parallel clasps (resolving a parallel clasp in a positive diagram reduces $l k$ by $4 n-1$ and $v_{2}$ by $n$ ). Thus let $D$ be a positive diagram of genus at most three with no clasps.

We fix a linked pair $(a, b)$ of crossings in $D$ and smooth them out, obtaining a genus-two diagram $D^{\prime}$. If $D$ cannot be simplified (after a possible sequence of flypes) by the inverse of a $\bar{t}_{2}$ move, we showed in the proof of Theorem 3.1 of [Sto01], that $D^{\prime}$ has at most four reducible crossings, but using the stronger condition that $D$ has no clasp, we see that our argument there modifies to show that in fact there is at most one reducible crossing $p$ in $D^{\prime}$. Let $D^{\prime \prime}$ be $D^{\prime}$ if $p$ does not exist, or the diagram obtained from $D^{\prime}$ after reducing $p$ according to (2), if $p$ exists.

It is easy to see that for knot diagrams with $p$ being the only reducible crossing on the left-hand side of (2), reducing $p$ never augments the number of clasps by more than one. ${ }^{1}$ Also, as smoothing out a crossing augments the number of clasps at most by two, $D^{\prime}$ has at most four clasps (note that one crossing may be in two clasps, as on the right-hand side of (15)).

Case 1. If $D^{\prime \prime}$ is connected and $p$ exists, then one of $P$ and $Q$ in (2) has no crossing. In this case, $a, b$ and $p$ are the corners of a triangular component of the complement of $D$, and smoothing out $b$ destroys (one of) the clasp(s) created by smoothing out $a$. Thus $D^{\prime}$ has (instead of at most four) in fact at most two clasps, and $D^{\prime \prime}$ has at most three clasps.

Case 2. If $D^{\prime \prime}$ is connected and $p$ does not exist, then $D^{\prime \prime}=D^{\prime}$ has at most four clasps, as noted.

Case 3. In the remaining case $D^{\prime \prime}$ has at most five clasps, but is disconnected (that is, the connected sum of two genus-one diagrams).

\footnotetext{
${ }^{1}$ Note that this is not true for link diagrams - consider the diagram of the Hopf link with one kink.
} 


\section{A. Stoimenow}

In all three cases the classification of genus-two diagrams of [Stob] shows that $D^{\prime \prime}$ has at most 13 crossings. Thus $D^{\prime}$ has at most 14 crossings, and $D$ has at most 16 crossings.

Now, the computer check of all less than or equal to 16 crossing diagrams without (reverse or parallel) clasp (up to flypes they are 203) shows that the ratio $l k / v_{2}$ on genus-three diagrams is indeed maximal on the mentioned 9 crossing diagram of 819 .

Now we use Lemma 5.2 to obtain an inequality between $v_{2}$ and $c$. To make the expressions somewhat simpler, instead of $3\lfloor(c-1) / 2\rfloor$ in the following we use $4 c / 3$, which is weaker except for $c \leqslant 8$ or $c=10,12,14,16$. For these crossing numbers $l k \geqslant 4 c / 3$ is checked directly by computer and is found to be true except for $c=3,4$, in which cases the subsequent claims are a matter of straightforward verification.

Therefore, from Proposition 5.1 we obtain the following.

Corollary 5.1. For any positive knot $K$ of genus at most three, we have $v_{2}(K) / c(K) \geqslant 20 / 63$.

To get from the positive to the almost positive case, we have the following lemma.

LEMma 5.3. If $D^{\prime}$ is an almost positive diagram that by flypes cannot be transformed into a diagram with (the negative crossing involved into) a resolved clasp, and $D$ is obtained from $D^{\prime}$ by switching the negative crossing to a positive one, then $v_{2}\left(D^{\prime}\right) \geqslant v_{2}(D)-l k(D) / 5$.

Proof. Let $p$ be the negative crossing in $D^{\prime}$. First we show that if $a \cap p$, then $\#\{q: q \cap a\} \geqslant 4$.

Assume the contrary, i.e. $\exists a \cap p: \exists ! c \neq p: c \cap a$. Now if $\exists d \cap c: d \not p$, then $2 C(d, c, a)$ would give a third chord intersecting $a$, and in the same way $2 C(d, p, a)$ will also do if $\exists d \cap p: d\lceil c$.

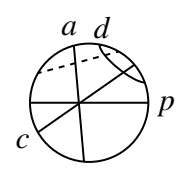

Therefore, $\{q: q \cap c\}=\{q: q \cap p\}$, which means that after possible flypes $p$ and $c$ form a (resolved) clasp.

Therefore, $\forall a \cap p: \#\{q: q \cap a\} \geqslant 4$. This implies that, if we set $v(p):=\#\{a: a \cap p\}$, then there are at least $3 v(p) / 2$ linked pairs in $D^{\prime}$ not involving $p$. The claim then follows from the fact (which is straightforward from the Gauß diagram sum formula), that if $D$ is the (positive) diagram obtained from $D^{\prime}$ by switching $p$, then $v_{2}(D)-v_{2}\left(D^{\prime}\right)=v(p) / 2$.

Corollary 5.2. For any almost positive knot $K$ of genus two, we have

$$
\frac{v_{2}(K)}{c(K)} \geqslant \frac{16}{315}
$$

Proof. An almost positive diagram $D$ of $K$ has genus at most three. Thus using Proposition 5.1 and Lemma 5.3, we get

$$
\frac{v_{2}(D)}{l k(D)} \geqslant \frac{5}{21}-\frac{1}{5}=\frac{4}{105}
$$

Multiplying the right-hand side by $4 / 3$ and replacing $l k(D)$ by $c(D)$ (by the weaker version of Lemma 5.2), and then $c(D)$ by $c(K)$, gives the result.

Note that we lose the genus-three case, as there may be almost positive genus-three knots that only possess almost positive diagrams of genus four. On the other hand, the statement for the genus-one case is obsolete, because almost positive genus-one knots do not exist (see [Stob]).

A presumably difficult combinatorial question that makes sense to ask now is the following. 

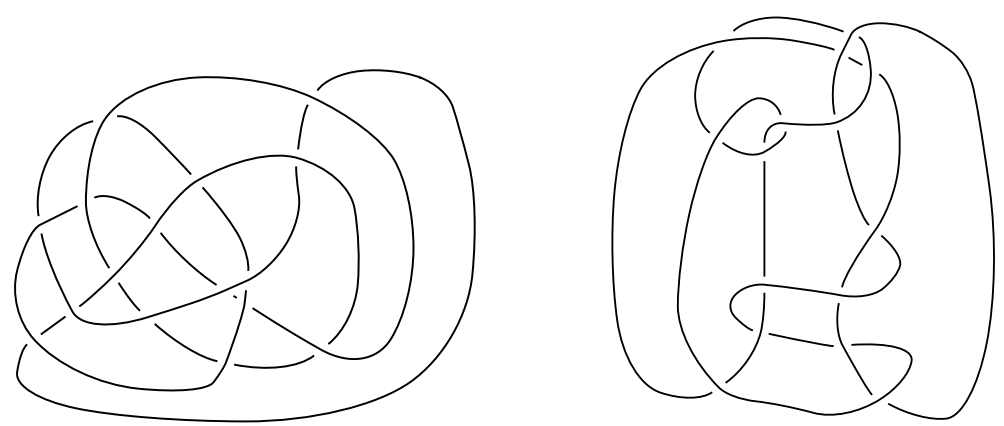

FiguRE 8: The 16 crossing diagram of the knot $15_{126448}$, maximizing the ratio $l k / v_{2}$ on all positive diagrams of at most 16 crossings, and the diagram of the same knot included in Thistlethwaite's table.

Question 5.1. Does $l k / v_{2}$ grow unboundedly on positive diagrams?

Remark 5.1. The computer experiment revealed that the maximal value of $l k / v_{2}$ on positive less than or equal to 16 crossing diagrams is attained on the diagram in Figure 8 of the $(4,5)$-torus knot $15_{126448}$, where it is $64 / 15=4.2 \overline{6}$. This, of course, is far from giving any indication about a (positive or negative) answer for Question 5.1.

Coming back to the case of arbitrary genus, we can obtain some partial improvements in the results of [Stoa] and those proved already in this paper.

Proposition 5.2. For any $\delta<3 / 8$ and any $g \in \mathbb{N}$, there are only finitely many positive knots of genus $g$ with $v_{2}<\delta c$.

Proof. Use Lemmas 5.2 and 5.1.

Proposition 5.3. For any $\delta<3 / 40$ and any $g \in \mathbb{N}$, there are only finitely many almost positive diagrams of almost positive knots of genus $g$ with $v_{2}<\delta$ c. In particular, there are only finitely many such knots.

Proof. By the extension of Bennequin's inequality [Ben83, Theorem 3] to arbitrary diagrams (see [Rud99, Stoa]), an almost positive knot of genus $g$ has almost positive diagrams only of genus $g$ or $g+1$, and thus it suffices to consider diagrams of bounded genus. It this situation Lemma 5.1 shows that, up to finitely many exceptions, on diagrams of given genus $v_{2}>(1 / 4-\varepsilon) l k$. Therefore, again up to finitely many exceptions, on almost positive diagrams of given genus, $v_{2}>(1 / 4-1 / 5-\varepsilon) l k$ by Lemma 5.3.

The above constant $3 / 40$ comes from the multiplication of $1 / 4-1 / 5=1 / 20$ by $3 / 2$, occurring in Lemma 5.2 (the defect constant in $c$ therein is put into the $\varepsilon$ ). Applying Lemma 5.2 we need to remark that if the positive diagram admits a move of the type (14), then so it does after the crossing change, unless then it looks like

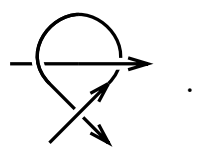

But in this case the negative crossing is linked with only two positive crossings, and therefore $v_{2}$ decreases only by one under the crossing change.

If we use the corollary for diagrams, we obtain the following. 


\section{A. Stoimenow}

COROllary 5.3. Each almost positive knot has only finitely many almost positive diagrams.

Proof. If there were infinitely many such diagrams, they would be of bounded genus, and then by Proposition 5.3, we would have $v_{2} \rightarrow \infty$ on them, a contradiction.

The next consequence is the main step towards the proof of Theorem 1.2.

Proposition 5.4. There are only finitely many almost positive knots with given $\max \operatorname{deg} V$ or $\max _{\operatorname{deg}_{v} P} P$ (in the variables of [CM92]). That is, if $K_{1}, K_{2}, \ldots$ is a sequence of distinct almost positive knots, then max $\operatorname{deg} V_{K_{i}} \rightarrow \infty$.

Proof. As for almost positive knots by [Stoc, Proposition 4.2] we have min $\operatorname{deg} V \geqslant g-1$; assume, taking a subsequence, that the genera of $K_{i}$ are bounded. Then by [Stod, Corollary 5.3] any coefficient of $V$ admits only finitely many values on $\left\{K_{i}\right\}$, and if $\max \operatorname{deg} V$ were bounded on $\left\{K_{i}\right\}$, so would be $v_{2}=-V^{\prime \prime}(1) / 6$, contradicting Proposition 5.3.

This argument establishes the desired property for a subsequence of $\left(K_{i}\right)$, but applying it to any subsequence of $\left(K_{i}\right)$ gives the result.

Exactly the same reasoning applies for $P$ (in fact, even for its absolute $[P]_{z^{0}}$ or quadratic terms $[P]_{z^{2}}$ of the Alexander variable, which contain $v_{2}$, see [KM98]).

Recall that the Thurston-Bennequin number $t b(\mathcal{K})$ of a Legendrian knot $\mathcal{K}$ in the standard contact space $\left(\mathbb{R}^{3}(x, y, z), d x+y d z\right)$ is the linking number of $\mathcal{K}$ with $\mathcal{K}^{\prime}$, where $\mathcal{K}^{\prime}$ is obtained from $\mathcal{K}$ by a push-forward along a vector field transverse to the (hyperplanes of the) contact structure.

The Maslov (rotation) index $\mu(\mathcal{K})$ of $\mathcal{K}$ is the degree of the map

$$
t \in S^{1} \mapsto \frac{\operatorname{pr} \frac{\partial \mathcal{K}}{\partial t}(t)}{\left|\operatorname{pr} \frac{\partial \mathcal{K}}{\partial t}(t)\right|} \in S^{1},
$$

where pr: $\mathbb{R}^{3} \rightarrow \mathbb{R}^{2} \simeq \mathbb{C}$ is the projection $(x, y, z) \mapsto(y, z)$.

Proof of Theorem 1.2. Combine the result of Proposition 5.4 for $P$ with the inequalities of [FT97, $\S 2$, Theorem 2.4] for $t b$ and $\mu$ coming from $\min \operatorname{deg}_{v} P$ (take care of the mirroring convention for the contact structure).

COROLlary 5.4. For any given genus $g$, there are only finitely many almost positive knots of genus $g$ with a given $\Delta$ or $Q$ polynomial.

Proof. Use again the fact that $v_{2}$ is contained in $\Delta$ and $Q$.

Remark 5.2. Of course, in view of the results on positive knots it is reasonable to conjecture the statement to be true even without the genus condition. For the Alexander polynomial it would follow from the following conjecture made in [Stoc].

Conjecture 5.2. If a knot $K$ is almost positive, then $\max \operatorname{deg} \Delta_{K}=\min \operatorname{deg} V_{K}$.

In [Stoa] we used the inequality of Theorem 6.1 therein to show that if a positive knot has a (reduced) positive diagram of $c$ crossings, then its crossing number is at least $\sqrt{2 c}$. This result can now be extended to almost positive knots, if we fix the genus.

COROLlary 5.5. Let $\delta<3 / 5$. Then for any genus $g$ there are only finitely many almost positive knots $K$ with a (reduced) almost positive diagram of $c$ crossings but $c(K)<\sqrt{\delta c}$.

Proof. Use again (the proof of) Proposition 5.3 and Theorem 1.E of [PV01]. The constant $3 / 5$ comes from multiplying the 1/20 appearing in the proof of Proposition 5.3 by the 8 in the denominator of Theorem 1.E of [PV01], giving $2 / 5$, and using the inequality $l k(D) \geqslant(3 / 2-\varepsilon) c(D)$ for a reduced diagram $D$. 


\section{GaUss DiAGRAM SUMS ON ALMOST POSITIVE KNOTS}

This result is indeed a little technical, but is exactly the missing piece required to extend the results of [Sto01] that the number of positive knots of given genus or unknotting number grows polynomially in the crossing number.

The proof of Theorem 1.1 is now analogous to the one in [Sto01].

Proof of Theorem 1.1. Consider first the genus case and fix $g$. Then, by Bennequin's inequality, we need to consider diagrams of genus at most $g+1$. Then Theorem 3.1 of [Sto01] shows that the number of such diagrams grows polynomially in the crossing number $c(D)$ of the diagram. But now, as we consider diagrams of bounded genus, we have by Corollary 5.5 only finitely many exceptions to throw out, to be allowed to apply, say, the inequality $c(K) \geqslant \sqrt{c(D) / 2}$, for $D$ a diagram of $K$. This shows that (up to these finitely many exceptions) we obtain all knots $K$ of given $c(K)$ by taking diagrams of at most $c(D) \leqslant 2 c(K)^{2}$ crossings, and a polynomial in $2 c(K)^{2}$ is a polynomial (of double degree) in $c(K)$.

The unknotting number result follows from that for the genus, because $u \geqslant g-1$ for an almost positive knot.

A final application of the methods described in this section is a simple proof of the following fact.

Proposition 5.5. There are only finitely many almost positive knots $K$ of given genus $g(K)$ and given braid index $b(K)$.

Proof. As a result of the braid index inequality

$$
b(K) \geqslant 1+\frac{1}{2} v-\operatorname{span}\left(P_{K}\right)=1+\frac{1}{2}\left(\max \operatorname{deg}_{v} P_{K}-\min _{\operatorname{deg}} P_{K}\right)
$$

of Franks and Williams [FW87] and Morton [Mor86], and Proposition 5.4, it suffices to show that for almost positive knots $K$ of given genus $g$, $\min \operatorname{deg}_{v} P_{K}$ is bounded (above). Now, it follows from the identity $P_{K}\left(v, v+v^{-1}\right)=1$ that $\min \operatorname{deg}_{v} P_{K} \leqslant \max _{\operatorname{deg}_{m}} P_{K}$, from an inequality of [Mor86] that $\max \operatorname{deg}_{m} P_{K} \leqslant 2 \tilde{g}(K)$, and from Bennequin's inequality that $\tilde{g}(K) \leqslant g(K)+1$ for an almost positive knot $K$. Joining all this, we obtain

$$
\min \operatorname{deg}_{v} P_{K} \leqslant \max \operatorname{deg}_{m} P_{K} \leqslant 2 \tilde{g}(K) \leqslant 2 g(K)+2,
$$

and thus the desired bound.

If desired, the estimates of the degrees of $V$ and $P$ can be made, in particular with regard to Theorem 1.2, more explicit. The lower bound we obtain for $\max \operatorname{deg}_{v} P(K)$ for $K$ positive or almost positive is of the form

$$
2 g(K)-2+\max \left(0, \sqrt[3]{A_{g(K)} c(K)-B_{g(K)}}\right),
$$

with certain constants $A_{g}>0$ and $B_{g}$ depending on $g$ only. $A_{g}$ will need to incorporate the constants of Propositions 5.2 and 5.3, and (in some exponential form) the numbers $d_{g}$ of [Sto01] as an estimate for the coefficients. The exceptional knots of Propositions 5.2 and 5.3 account for the correction term $B_{g}$. In (17), the cube root term comes from the $v$-span $n$ of $X(v)=[P(K)]_{z^{0}}$, and is explained as follows: when min $\operatorname{deg}_{v} X \leqslant 2 g$ and $X \in \mathbb{Z}\left[v^{2}\right]$, then $X$ has at most $n / 2+1$ non-zero coefficients bounded by $C_{g}$, and the derivation produces a factor of at most $(2 g+n)(2 g+n-1)$ for the coefficient of each monomial, so that

$$
v_{2}=C\left|X^{\prime \prime}(1)\right| \leqslant(2 g+n)^{2} \cdot(n / 2+1) \cdot C_{g} \leqslant C_{g}^{\prime} \cdot n^{3},
$$

for certain constants $C, C_{g}$ and $C_{g}^{\prime}$. This cube root thus also gives an estimate for the braid index of $K$ via (16). See also [Sto99], where similar degree estimates for $V$ and for positive knots are given. 


\section{A. Stoimenow}
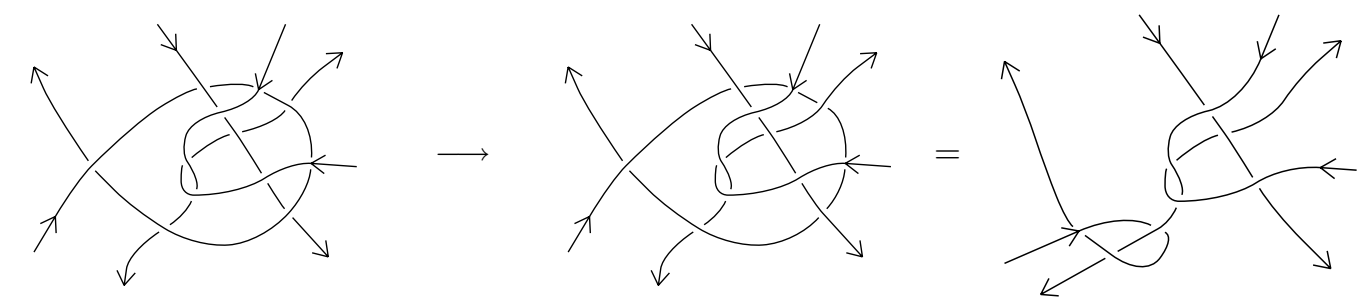

Figure 9: An almost loop-move. Take a crossing whose smoothing out gives a component with no self-crossings, and switch some of the crossings on the segment except the last one, so that the segment to (over-/under-)pass them all in different way than the last one. Then the segment can be simplified to have just two crossings on it.

\section{The signature}

Although the Vassiliev invariant inequalities for positive knots of [Stoa] have nice theoretical consequences, they are revealed to be too weak to exclude knots with almost positive diagrams from being positive. In this regard the most handy criterion is obtained using the signature $\sigma$. It was announced by Przytycki and Taniyama in [PT91], but a proof was never published. We give an independent proof of this criterion, using some methods and results of [Stoa], [Sto01] and [Stob], and then apply it to show that there are infinitely many almost positive knots.

Theorem 6.1. (See [PT91]). Any positive knot has signature of at least 4, except for the $(p, q, r)$-pretzel knots with $p, q, r>0$ odd, which have signature 2.

Proof. In [Stoa, § 6] we introduced a move on (positive) diagrams, called loop-move, to show an inequality of the Casson invariant (Theorem 6.1) and observed that it can also be used to show the positivity of the signature, a result of Cochran and Gompf [CG88, Corollary 3.4, p. 497] and Traczyk [Tra88].

The loop-move from a diagram $A$ to a diagram $B$, henceforth denoted by $A \rightarrow B$, consists of choosing a segment of the line in $A$ between the two passings of a crossing, such that it has no selfcrossings, and removing this segment by switching half of the crossings on it (and elimination of all reducible crossings thereafter). Such a move never augments the signature, that is, $\sigma(B) \leqslant \sigma(A)$, and a finite number of such moves makes any positive diagram trivial, that is, for any diagram $D$, there is a sequence $D=D_{0} \rightarrow D_{1} \rightarrow \cdots \rightarrow D_{n}$ of loop moves, with $D_{n}$ being the zero crossing diagram.

By considering a loop move sequence in which $n$ is maximal, the diagram $D^{\prime}:=D_{n-1}$ before applying the last step of such a sequence can be chosen so that any loop move unknots it. If $\sigma\left(D^{\prime}\right) \geqslant 4$, then the assertion is satisfied. By [Stoa, Exercise 6.4] and [Sto01] $\sigma\left(D^{\prime}\right)=2$ exactly if $D^{\prime}$ is a diagram of genus one, in which case it corresponds to one of the desired pretzel knots.

Therefore, it remains to show that if $D^{\prime}$ is of genus one, but $n>1$, then $\sigma(D) \geqslant 4$. We show that already $\sigma\left(D^{\prime \prime}\right) \geqslant 4$ for $D^{\prime \prime}:=D_{n-2}$.

Replace the loop move $D^{\prime \prime} \rightarrow D^{\prime}$ by an almost loop-move, as shown in Figure 9. One obtains from $D^{\prime \prime}$ a (positive) diagram $\tilde{D}$, from which $D^{\prime}$ arises by switching on of the two crossings of the segment removed by $D^{\prime \prime} \rightarrow D^{\prime}$. By direct observation one sees that (independently of the orientation), the move $\tilde{D} \rightarrow D^{\prime}$ (which is formally also a loop-move), preserves the number of Seifert circles, and hence $\tilde{D}$ has genus two.

But all (inter alia, positive) genus-two diagrams are classified in [Stob], and are in particular shown to be transformable by changing positive crossings to certain 24 (called therein 'generating') diagrams, and it is straightforward to check that for all of them $\sigma \geqslant 4$ (see Corollary 3.2 therein), which shows the assertion. 


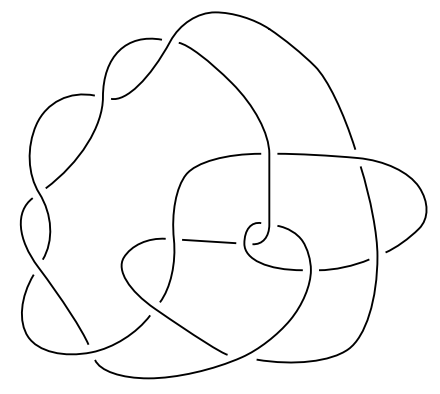

Figure 10 . The knot $! 14_{34605}$, a member of an infinite family of almost positive knots.

Remark 6.1. When the length $n$ of the loop-move sequence becomes large, then it is apparent that a much better estimate should be obtainable for the signature. This evidence is compatible with the conjecture made in [Sto00] that $\sigma$ is bounded below by an increasing function of the genus for positive (and hence also for almost positive) knots. To prove such a statement using loop-moves only seems difficult, though. Although a loop move in general reduces $\sigma$, this need not always be the case. Worse yet, there are positive diagrams, on which none of the applicable loop-moves strictly reduces $\sigma$ (one such diagram is the 14 crossing diagram of the knot $14_{45657}$ given in [Sto00]).

Example 6.1. There are infinitely many almost positive knots. Consider the knot ! $14_{34605}$ of Hoste and Thistlethwaite [HTW98, HT] in Figure 10. It is a genus-two knot of determinant -3 , and hence has signature 2. Switching one of the five crossings forming reverse clasps in the upper left-hand side of the diagram, we obtain ! $122_{1692}$, a knot with the same genus and determinant, and also the same for $! 10_{145}$, obtained by switching two of these five crossings. Therefore, applying $\bar{t}_{2}$ moves at the five crossings

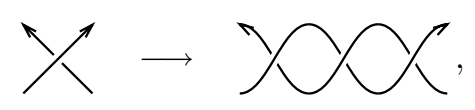

we obtain an infinite family of knots starting with $! 10_{145}, ! 12_{1692}, ! 14_{34605}, ! 16_{970714}, \ldots$ with genus two and determinant -3 , and hence signature 2 . If some of them were positive, then it would have to be a pretzel knot, and would have genus one. On the other hand, the diagrams are evidently all almost positive, therefore so are all these knots. Moreover, the diagrams obtained are indeed of minimal crossing number as can be shown by examining their $Q$ polynomial [Kid87] and using the fact that by [Stoc, $\S 5]$ the knots are non-alternating.

\section{Corollary 6.1. Any almost positive knot has positive signature.}

Proof. Switch the one negative crossing to the positive, which does not augment $\sigma$ by more than 2, and apply the previous theorem. The only point to remark is that an almost positive diagram of genus one corresponds to a positive knot.

Remark 6.2. In fact, it is worth mentioning that the loop-move can also be used to show Taniyama's theorems of [Tan89], that any non-trivial knot diagram can be crossing-switched to a diagram of the trefoil, and that any connected knot diagram of genus at least two can be done so to the $(5,2)$-torus knot. For this we just need not to pull out the loops in the loop-moves and to switch any subsequent loop above this additional set of crossings (remaining by not pulling out the previous loops). If we need to perform an almost loop-move in the end, then we switch the crossings on this loop (except the one) so as to pull the loop below the rest of the diagram. Then we need to consider only the 24 generating genus-two diagrams of [Stob, Figure 5] (modulo flypes, which are irrelevant here), for which the claims can be verified directly. 


\section{A. Stoimenow}

\section{A conjecture}

The considerations open a natural question about further generalizations.

Definition 7.1. A knot is called 2-almost positive, if the minimal number of negative crossings in all its diagrams is 2 .

Although it is not clear how the classification result should carry over to 2-almost positive unknot diagrams, at a first glance it appears that at least Theorem 2.1 should generalize to this case. Surprisingly, this turns out not to be the case.

Example 7.1. The knots $! 6_{1}$ and $! 6_{2}$ have in their (2-almost positive) 6 crossing diagrams $v_{3}=-4$. This also shows that $! 6_{1}$ and $! 6_{2}$ are indeed 2-almost positive. Furthermore, as $! 6_{1}$ and $! 6_{2}$ can be unknotted in their 6 crossing diagrams by switching only positive crossings, this shows that (although measuring positivity in general by positive values, contrarily to the signature) $v_{3}$ increases sometimes, when a positive crossing is switched to a negative one.

Therefore, unfortunately, our approach is very unlikely to carry over to classify 2-almost positive unknot diagrams. This has been achieved in [Stob] using a deeper tool - the version of the inequality of Bennequin for arbitrary knot diagrams [Rud99, Stoa].

However, with some heuristics the above example leads to the following conjecture.

Conjecture 7.1. Let $D$ be a 2 -almost positive even crossing number diagram minimizing $v_{3}$ over all diagrams of that crossing number. Then $D$ is a diagram of a $\left(a_{1}, \ldots, a_{k}, 2\right)$ pretzel knot, $a_{i} \in\{1,3\}$.

\section{ACKNOWLEDGEMENTS}

I would like to thank the referee for his useful advice. I would also like to thank V. Chernov for some helpful discussions and for his invitation to Zürich in the summer of 1999. Finally, I am very grateful to the Editors of Compositio Mathematica for giving me the opportunity to publish this paper in their journal, after being rejected nine times over the course of five years since its initial writing.

\section{REFERENCES}

Ada92 C. C. Adams, J. F. Brock, J. Bugbee, T. D. Comar, K. A. Faigin, A. M. Huston, A. M. Joseph and D. Pesikoff, Almost alternating links, Topol. Appl. 46 (1992), 151-165.

Ada94 C. C. Adams, Das Knotenbuch (Spektrum Akademischer Verlag, Berlin, 1995) The knot book (Freeman, New York, 1994).

Ale28 J. W. Alexander, Topological invariants of knots and links, Trans. Amer. Math. Soc. 30 (1928), $275-306$.

AM90 S. Akbulut and J. D. McCarthy, Casson's invariant for oriented 3-spheres, Mathematical Notes, vol. 36 (Princeton, 1990).

Bar95 D. Bar-Natan, On the Vassiliev knot invariants, Topology 34 (1995), 423-472.

Bar D. Bar-Natan, Bibliography of Vassiliev invariants, available from the web site http://www.math.toronto.edu/drorbn/VasBib/VasBib.html.

Ben83 D. Bennequin, Entrelacements et équations de Pfaff, Soc. Math. France, Astérisque 107-108 (1983), 87-161.

BLM86 R. D. Brandt, W. B. R. Lickorish and K. Millett, A polynomial invariant for unoriented knots and links, Inv. Math. 84 (1986), 563-573.

BM71 R. Bing and J. Martin, Cubes with knotted holes, Trans. Amer. Math. Soc. 155 (1971), 217-231.

BS88 S. Bleiler and M. Scharlemann, A projective plane in $\mathbb{R}^{4}$ with three critical points is standard. Strongly invertible knots have property P, Topology 27(4) (1988), 519-540.

CG88 T. D. Cochran and Robert E. Gompf, Applications of Donaldson's theorems to classical knot concordance, homology 3-spheres and Property P, Topology 27(4) (1988), 495-512. 


\section{GAUSS DIAGRAM SUMS ON ALmost POSITIVE KNOTS}

CGM00 S. Chmutov, V. Goryunov and H. Murakami, Regular Legendrian knots and the HOMFLY polynomial of immersed plane curves, Math. Ann. 317(3) (2000), 389-413.

CM92 P. R. Cromwell and H. R. Morton, Positivity of knot polynomials on positive links, J. Knot Theory Ramif. 1 (1992), 203-206.

Con69 J. H. Conway, On enumeration of knots and links, in Computational problems in abstract algebra, ed. J. Leech (Pergamon Press, 1969), 329-358.

Cro89 P. R. Cromwell, Homogeneous links, J. London Math. Soc. (Series 2$) 39$ (1989), 535-552.

DL01 O. T. Dasbach and X.-S. Lin, The Bennequin number of $n$-trivial closed n-braids is negative, Math. Res. Lett. 8(5-6) (2001), 629-635.

DM01 O. T. Dasbach and B. S. Mangum, On McMullen's and other inequalities for the Thurston norm of link complements, Algebr. Geom. Topol. 1 (2001), 321-347.

DR99 C. Delman and R. Roberts, Alternating knots satisfy strong property P, Comment. Math. Helv. 74(3) (1999), 376-397.

FHL85 P. Freyd, J. Hoste, W. B. R. Lickorish, K. Millett, A. Ocneanu and D. Yetter, A new polynomial invariant of knots and links, Bull. Amer. Math. Soc. 12 (1985), 239-246.

Fie93 T. Fiedler, A small state sum for knots, Topology 32(2) (1993), 281-294.

Fie01 T. Fiedler, Gauss sum invariants for knots and links, Mathematics and Its Applications, vol. 532 (Kluwer Academic, Dordrecht, 2001).

FS00 T. Fiedler and A. Stoimenow, New knot and link invariants, in Proc. int. conf. knot theory 'Knots in Hellas, 98', Series on knots and everything, vol. 24 (World Scientific, Singapore, 2000).

FT97 D. Fuchs and S. Tabachnikov, Invariants of Legendrian and transverse knots in the standard contact space, Topology 36(5) (1997), 1025-1053.

FW87 J. Franks and R. F. Williams, Braids and the Jones-Conway polynomial, Trans. Amer. Math. Soc. 303 (1987), 97-108.

Gab86 D. Gabai, Genera of the alternating links, Duke Math. J. 53(3) (1986), 677-681.

Hak61 W. Haken, Theorie der Normalflächen, Acta Math. 105 (1961), 245-375.

Hir00 M. Hirasawa, Triviality and splittability of special almost alternating links via canonical Seifert surfaces, Topol. Appl. 102(1) (2000), 89-100.

HL01 J. Hass and J. C. Lagarias, The number of Reidemeister moves needed for unknotting, J. Amer. Math. Soc. 14(2) (2001), 399-428.

Ho85 C. F. Ho, A polynomial invariant for knots and links - preliminary report, Abstracts Amer. Math. Soc. 6 (1985), 300.

HT J. Hoste and M. Thistlethwaite, KnotScape, a knot polynomial calculation program, available at http://www.math.utk.edu/ morwen.

HTW98 J. Hoste, M. Thistlethwaite and J. Weeks, The first 1,701,936 knots, Math. Intell. 20(4) (1998), 33-48.

JLWW02 B. Jiang, X.-S. Lin, S. Wang and Y.-Q. Wu, Achirality of knots and links, Topology Appl. 119(2) (2002), 185-208.

Jon85 V. F. R. Jones, A polynomial invariant of knots and links via von Neumann algebras, Bull. Amer. Math. Soc. 12 (1985), 103-111.

Jon87 V. F. R. Jones, Hecke algebra representations of braid groups and link polynomials, Ann. Math. 126 (1987), 335-388.

Kan88 T. Kanenobu, An evaluation of the first derivative of the $Q$ polynomial of a link, Kobe J. Math. 5 (1988), 179-184.

Kan98 Y. Kanda, On the Thurston-Bennequin invariant of Legendrian knots and nonexactness of Bennequin's inequality, Invent. Math. 133(2) (1998), 227-242.

KM98 T. Kanenobu and Y. Miyazawa, HOMFLY polynomials as Vassiliev link invariants, in Knot theory, Banach Center Publications, vol. 42, eds V. F. R. Jones, J. Kania-Bartoszyńska, J. H. Przytycki, P. Traczyk and V. G. Turaev (Banach Center Publications, Warszawa, 1998), 165-185. 


\section{GAUss DIAGRAM SUMS ON ALmOST POSITIVE KNOTS}

Kau90 L. H. Kauffman, An invariant of regular isotopy, Trans. Amer. Math. Soc. 318 (1990), $417-471$.

Kid87 M. Kidwell, On the degree of the Brandt-Lickorish-Millett-Ho polynomial of a link, Proc. Amer. Math. Soc. 100 (1987), 755-761.

KL98 E. Kalfagianni and X.-S. Lin, Regular Seifert surfaces and Vassiliev knot invariants, math/9804032.

KSA97 A. Kricker, B. Spence and I. Aitchison, Cabling the Vassiliev invariants, J. Knot Theory Ramif. 6 (1997), 327-358. See also q-alg/9511024.

LT88 W. B. R. Lickorish and M. B. Thistlethwaite, Some links with non-trivial polynomials and their crossing numbers, Comment. Math. Helv. 63 (1988), 527-539.

Lin94 X.-S. Lin, Finite type link invariants of 3-manifolds, Topology 33(1) (1994), 45-71.

MR01 M. McDaniel and Y. Rong, Vassiliev invariants from satellites of link polynomials, Kobe J. Math. 18(2) (2001), 127-145.

MT91 W. W. Menasco and M. B. Thistlethwaite, The Tait flyping conjecture, Bull. Amer. Math. Soc. 25(2) (1991), 403-412.

MZ00 W. W. Menasco and X. Zhang, Positive knots and knots with braid index three have property P, math.GT/0010154, to appear in J. Knot Theory Ramif.

Mor86 H. R. Morton, Seifert circles and knot polynomials, Proc. Camb. Phil. Soc. 99 (1986), $107-109$.

Mur87 K. Murasugi, Jones polynomial and classical conjectures in knot theory, Topology 26 (1987), 187-194.

PT91 J. Przytycki and K. Taniyama, Almost positive links have negative signature, unpublished draft. Abstr. Amer. Math. Soc. 12(3) (1991), 327.

PV94 M. Polyak and O. Viro, Gauss diagram formulas for Vassiliev invariants, Int. Math. Res. Notes 11 (1994), 445-454.

PV01 M. Polyak and O. Viro, On the Casson knot invariant, Knots in Hellas '98, vol. 3 (Delphi), J. Knot Theory Ramif. 10(5) (2001), 711-738.

Rol76 D. Rolfsen, Knots and links (Publish or Perish, Berkeley, CA, 1976).

Rud90 L. Rudolph, A congruence between link polynomials, Math. Proc. Camb. Phil. Soc. 107 (1990), 319-327.

Rud99 L. Rudolph, Positive links are strongly quasipositive, Proceedings of the Kirbyfest, Geometry Topol. Monographs 2 (1999), 555-562. See also http://www.maths.warwick.ac.uk/gt/GTMon2/paper25.abs.html.

Stoa A. Stoimenow, Positive knots, closed braids, and the Jones polynomial, math/9805078, to appear in Ann. Scuola Norm. Sup. Pisa Cl. Sci.

Stob A. Stoimenow, Knots of genus two, Preprint math.GT/0303012.

Stoc A. Stoimenow, On some restrictions to the values of the Jones polynomial, Preprint.

Stod A. Stoimenow, Polynomial and polynomially growing knot invariants, Preprint.

Stoe A. Stoimenow, A property of the skein polynomial with an application to contact geometry, Preprint math.GT/0008126.

Sto99 A. Stoimenow, Jones polynomial, genus and weak genus of a knot, Ann. Fac. Sci. Toulouse VIII(4) (1999), 677-693.

Sto00 A. Stoimenow, The signature of 2-almost positive knots, J. Knot Theory Ramif. 9(6) (2000), 813-845.

Sto01 A. Stoimenow, Knots of genus one, Proc. Amer. Math. Soc. 129(7) (2001), 2141-2156.

Tan89 K. Taniyama, A partial order of knots, Tokyo J. Math. 12(1) (1989), 205-229.

Tra88 P. Traczyk, Non-trivial negative links have positive signature, Manuscripta Math. 61 (1988), 279-284.

Vas90 V. A. Vassiliev, Cohomology of knot spaces, in Theory of singularities and its applications, ed V. I. Arnold (American Mathematical Society, Providence, RI, 1990).

A. Stoimenow stoimeno@math.fu-berlin.de

Warnitzer Str. 19, 13057 Berlin, Germany 